\title{
ZU TRAUTMANN'S DEUTUNG DES ERSTEN UND NEUNUNDACHTZIGSTEN RÄTSELS.
}

Trautmann bat anzeiger zur Anglia VI, s. $158 \mathrm{ff}$. und anzeiger zur Anglia VII, s. 210 nachzuweisen versucht, dass die bisherigen auflösungen des 1. und 89. rätsels zu verwerfen und dafür die deutung 'das rätsel' für beide anzuerkennen sei. Mir scheint jedoch, dass Trautmann's lösung auch nicht den geringsten vorzug vor der fruheren verdient. Was er an der von Leo gegebenen auflösung zu rätsel I tadelt, ist auch von anderer seite längst als verbesserungsbedürftig anerkannt und geändert worden. Ich verweise nur auf den aufsatz von Rieger, Ztschr. f. d. Ph. I, $215 \mathrm{ff}$, der mir in vielen punkten das richtige getroffen zu haben scheint; ferner auf Dietrich, Haupt's Ztschr. XI, 458, und auf Wulker, Anglia I, 493. Ich bin weit davon entfernt, diese deutungsversuche als völlig befriedigende hinzustellen; sie muten aber jedenfalls dem ratenden viel weniger spitzfindige kombinationsgabe $\mathrm{zu}$, als die von Trautmann gegebenen, der jedem satze der beiden rätsel zwei bis drei andere hinzufugt, um dem leser klar zu machen, was er in den urspriglichen satz hineinlesen will.

Ich werde nun Trantmann's ausfuhrungen näher besprechen.

Er betrachtet s. 164 zunächst vv. 4-17 des ersten rätsels: Wulf is on îege, ic on opperre = 'der wolf ist auf einer insel, ich auf der anderen'. Dazu gibt Trautmann die erläuterung: 'Der wolf (der mich zu erbenten sucht, der ratende) ist auf einer insel; ich, das rätsel, bin auf einer anderen (d. i. rätsel und ratender sind getrennt von einander)'. Dagegen habe ich einzuwenden: Der wolf, als der ratende, erbeutet schliesslich nicht das rätsel, sondern die lösung desselben, wie Trautmann selbst $\nabla .17$ deutet. Ferner sind das rätsel und der ratende nicht von einander getrennt; das wäre ein total verfehltes bild. 
Nur die lösung des rätsels und der ratende können als von einander getrennt vorgestellt werden.

V. 6: sindon wel-hreove weras pher on îge. Dazu sagt Trautmann: 'Auf jener insel (wo der wolf ist) sind kampfwilde männer (d. i. es sind beim wolfe, bei dem, der mich schliesslich errät, noch andere, die mich zu erraten suchen, das rätsel wird in einer gesellschaft aufgegeben)'. Dies alles liest Trautmann aus einer zeile heraus, und dabei macht er Leo den vorwurf, in das rätsel etwas hineingelesen zu haben, was nicht darin enthalten sei. Ungeschickter und unklarer hätte der dichter sich nicht ausdriicken können, wenn er das hätte sagen wollen, was Trautmann ihm unterschiebt. Es steht ferner nicht da: 'auf jener insel, wo der wolf ist'; ausserdem aber würde der dichter wol nicht die ratende gesellschaft aus einem wolf und kampfwilden männern haben bestehen lassen, das wäre doch eine zu sonderbare zusammenstellung.

V. 9: wulles ic mines widlâslum wênum dogode = 'ich war betrübt über meines Wulf weitgreifendes wähnen'. wên aber müsste erst als 'wähnen' nachgewiesen werden; wenn dies aber auch wirklich gelänge, so würde doch wol niemand diesen worten den sinn unterlegen: 'Ich war betrubt, weil Wolf weit umherriet, ohne meine lösung zu finden', wie Trautmann von uns verlangt.

V. 10: ponne hit was renig weder ond ic reotugu sat ubersetzt und deutet Tr.: 'Dann war regenwetter [für mich], und ich sass und weinte'. Wo steht denn aber 'für mich'? Diese beiden worte hïtten nicht fehlen diirfen, wenn der dichter den anfang des satzes haitte bildlich fassen wollen, wie Tr. ihn nimmt.

V. 11: ponne mec se beadu-cr̂fa bogum bilegde = 'wenn mich der kampfschnelle mit seinen armen umschloss'. Das soll heissen: 'wenn mich der wolf erriet'. Damit hätte aber der dichter das bild mit dem wolfe als ratendem verlassen, um es schon im näichsten verse wider aufzunehmen. Die worte: 'wenn mich der kampfschnelle mit seinen armen umschloss' würde wol tibrigens auch niemand als: 'wenn er mich, das rätsel, erriet' verstehen.

Doch weiter; ich werde jetzt nur die ibersetzung Tr.'s geben und das, was er in sie hineinlegt: 'Wolf, mein wolf! Dein wähnen (soll nach ihm heissen: dein falsches raten), dein 
seltenes kommen (darunter sollen wir verstehen: dass du der auflösung so selten nahe kamst!) haben mich krank gemacht'! Das soll ein dichter von einem rätsel haben sagen lassen, und wir sollen es verstehen!

V. 16 und 17: 'Hörst du, Eadwacer, unser beider muntern welf, schleppt der wolf znm walde!' Dazu sagt Trautmaun (s. 165): 'Jeder rechtschaffene wolf wird seine beute in den wald schleppen. Wie aber könnte der dichter den gedanken "der ranb ist vollzogen", d. i. "das rätsel ist gelöst", zierlicher ausdrücken als durch die wendung: "hörst du? der wolf schleppt seinen raub in's holz"?' Es wird uns also zugemutet, unter Eadwacer, dem munteren hlindchen, die lösung zu verstehen, seine eltern sollen das rätsel und der wolf sein. Das ist doch jedenfalls ein wenig zu viel von dem ratenden verlangt, und man frïgt sich unwillkürlich dabei, wie $\mathrm{Tr}$. auf diese sonderbare anslegung der vv. 16 und 17 gekommen ist. Bei dieser stelle hat jedenfalls Rieger, Ztschr. f. d. Ph. I, 215 ff. das richtige getroffen.

Wir wenden uns nun zu dem, was Tr. aus anfang und ende des ersten rätsels machen will. Er ubersetzt den anfang: 'Es ist meinen leuten, als ob ihnen jemand gaben bringe; sie wollen ihn aufnehmen, wenn er zu ihnen kommt'. Natürlich würde dies kein mensch als von einem rätsel gesagt auffassen. Um dergleichen zu vermuten muss man notwendigerweise Tr.'s erlänterungen zur seite haben. Hinter 'leuten' setzt er in klammern: '(d. i. uns rätseln)'. Dem übrigen fiigt er die erläuterung hinzu: 'Wie man jemand, der geschenke bringt, aufnimmt, nicht abweist, so nehmen auch wir rätsel den auf, bekennen uns dem als gelöst, der uns errät; wie es jedem angenehm ist, geschenke zu erhalten, so ist es uns rätseln angenehm, erraten zu werden'. Wie man dies alles ans den zwei zeilen herauslesen kann, ist für mich auch ein rätsel! Ausserdem aber scheint Trautmann dabei vergessen zu haben, dass seiner ansicht nach die lösung in vv. 16/17 als ein raub dargestellt ist, während hier in den anfangsversen das rätsel die lösung als ein geschenk des ratenden bezeichnet. Ueber die bedeutung des schlusses ist Trantmann nicht mit sich im klaren, so dass wir dartber wol hinweggehen durfen.

Was die bemerkungen zur erläuterung und rechtfertigung von Trautmann's deutung auf seite 165 angeht, so hätte er 
sich dieselben vollkommen ersparen können, sie beweisen garnichts.

Wir kommen nun zum zweiten teil von Tr.'s arbeit, der deutung von rätsel no. 89. Ich bin auch hier der ansicht, dass Dietrich mit der alten lösung das richtige getroffen hat, und dass wir gut tun, bei ihr zn verharren.

Ich werde hier zunächst die ubersetzung des rätsels geben, wie Trautmann sie wünscht, sein nachtrag im anzeiger zur Anglia VII, s. 210 ist dabei berticksichtigt:

Ich bin edel und edelen männern bekannt

Und weile oft von hohen und niederen

Leuten geehrt. Weithin fährt,

Verbreitet sich die umarmung des fremdlings,

Die lust der plïnderer, und sie steht mir vor den freunden,

Wenn ich glïck und glänzendes gut in den burgen haben soll.

Kluge leute lieben sehr

Meine gesellschaft; ich soll vielen

Weisheit küinden, sie sprechen dabei kein wörtchen.

Obwol nun die kinder der menschen

Der landbebautenden meine stapfen

Eifrig suchen, verberge ich doch zuweilen

Jedem der männer meine spur'.

Dies ist die tibersetzung, wie sie Trautmann sich zurechtgelegt bat, um die deutung ' $\mathrm{das}$ rätsel' herauszubekommen. Meiner ansicht nach aber kann kein mensch aus diesen dreizehn zeilen herauslesen, dass ein rätsel von sich spricht, es sei denn, dass zwischen den zeilen alle jene erläuterungen ständen, die Trautmann sich genötigt sieht hinzuzufugen.

Den ersten satz umschreibt er mit den worten: 'Ich werde oft bei hohen und niedrigen aufgegeben (sic!) und hohe und niedrige kennen und ehren mich'. Ja, auf das wort 'aufgeben' kommt alles an; ohne dies wort, das nicht im texte steht, wird kein mensch an ein rïtsel denken. Zum zweiten satz sagt er: 'Durch den ganzen saal, die ganze gesellschaft geht die freude der ratenden, sobald ich aufgegeben werde; und das nmherraten der fremden, derer, die meine bedeutung noch nicht kennen, und die wonne der plunderer, derer die mich zu erraten trachten oder erraten, muss mir lieber sein als die freunde, als die, welche meine ledeutung bereits wissen oder mich anfgeben, wenn ich in den burgen beifall finden und lohn ernten soll'. Das alles bat Tr. in den einen satz hineingelesen! So aber, wie Tr. die ubersetzung gibt, wurde, glaube 
ich, jeder eher denken, es bandle sich um einen Wickinger, der auf beute ausfährt und dem die lust am plundern, am frendigen seekrieg, der ihm schätze in fulle verschafft, vor dem verkehr mit den freunden steht, als dass ein rätsel von sich spricht! Den dritten satz, zeile 7-9, könnte ebenso gut oder vielmehr noch besser ein professor von seinen zuhörern sagen als ein rätsel, denn ein solches verktundet doch keine weisheit, im gegenteil, es verlangt weisheit von dem ratenden. Den letzten satz endlich hält Trautmann jedenfalls für den beweiskräftigsten; durch ihn hat er sich wol zu dem ganzen unglucklichen deutungsversuch hinreissen lassen. Er erklärt ihn durch die worte: 'Trotz angestrengten nachsinnens erraten mich doch manchmal die leute nicht'. Hätte aber wol der dichter das rätsel seine lösung als 'meine stapfen' bezeichnen lassen? Schwerlich, er hätte es von seinem kern, inhalt oder derartigem reden lassen, nicht aber von 'spuren'. Ausserdem aber wlurde das rätsel mit monna gehwylcum eine grobe unwahrheit aussprechen, denn den menschen verdankt es ja uberhaupt nur sein dasein!

Ich will mich nicht einlassen auf die konjektur, die Trautmann im anzeiger zur Anglia VII zu v. 4 gibt, welche, an sich betrachtet, als eine ganz glückliche bezeichnet werden muss. Sie ist aber unntitz, denn die lösung 'das rätsel' ist nicht die richtige, und nur für sie passt die konjektur!

BERLIN.

R. NUCK. 